\title{
Vasodilator myocardial perfusion imaging: demonstration of local electrophysiological changes of ischaemia
}

Roy M John, Peter I Taggart, Peter M Sutton, Durval C Costa, Peter J Ell, Howard Swanton

\begin{abstract}
Objective-To examine the incidence and severity of myocardial ischaemia provoked in the course of perfusion scintigraphy by coronary vasodilators using endocardial recordings of steady state monophasic action potentials as an independent marker of early localised myocardial ischaemia.
\end{abstract}

Patients-31 men undergoing routine cardiac catheterisation for investigation of chest pain were studied.

Setting-A tertiary cardiac referral centre.

Design-Single site monophasic action potentials were recorded from the left or right ventricle or both (50 recording sites) during intravenous infusion of dipyridamole $(0.015 \mathrm{mg} / \mathrm{kg} / \mathrm{min})$ for four minutes. Heart rate was held constant with atrial pacing at $20 \%$ above the patient's resting rate. Technetium-99m hexakis-2-methoxy-2-methylpropyl-isonitrile (MIBI) was administered four minutes after dipyridamole, and single photon emission tomographic imaging was performed an hour later. Rest images were obtained the next day (two day, two dose protocol). Recordings were divided into three groups based on the scintigraphic perfusion characteristics and coronary anatomical data for the action potential recording site: group 1recordings from areas with a normal perfusion pattern $(n=30)$, group 2 recordings from areas with a perfusion defect and subtended by significantly narrowed coronary arteries without obvious angiographic collateral supply $(n=10)$, and group 3-recordings from areas with a perfusion defect and subtended by occluded arteries with angiographically evident collaterals from adjacent vessels $(n=10)$.

Results-There were changes in the duration of the monophasic action potential indicative of ischaemia-that is, shortening of duration of steady state action potential-in 18 of the 20 recordings from areas of abnormal perfusion. Peak changes were apparent eight minutes from the start of the dipyridamole infusion. Mean (SEM) values for duration of the action potential between control and peak effect at eight minutes were
$276.5(5 \cdot 3) \mathrm{ms} v 276.6(5.4)$ for group 1 (NS), $289.6(4.7) \mathrm{ms} v 278.4(4.9) \mathrm{ms}$ for group $2(p<0.001)$, and $269.6(5.7) \mathrm{ms} v$ $242.0(4.4)$ for group $3(p<0.0001)$. These changes were significantly different between the three groups $(p<0.01)$. ST segment changes on the surface electrocardiogram were seen in only eight patients, all with areas of viable myocardium supplied by collateral vessels.

Conclusions-These data provide strong evidence for the presence of myocardial ischaemia in regions of reversible perfusion defects induced by dipyridamole. This study also shows that such ischaemia is more intense and more likely to be seen when myocardial viability is dependent on collateral circulation.

Myocardial perfusion scintigraphy using vasodilators such as dipyridamole or adenosine is a widespread diagnostic tool although the underlying pathophysiological mechanisms remain incompletely defined. ${ }^{1-3}$ Both dipyridamole and adenosine dilate coronary arteries and produce a substantial increase in coronary blood flow. ${ }^{45}$ In coronary vascular beds distal to a flow limiting stenosis, autoregulatory mechanisms operate producing maximal vasodilatation under resting conditions and thereby limiting coronary flow reserve. ${ }^{6}$ Dipyridamole, for example, would therefore induce a preferential dilatation in the vascular bed subtended by normal arteries but exert minimal effects on those distal to a stenotic artery. ${ }^{5}$ These regional differences in blood flow would unevenly distribute added radiotracers, producing scintigraphic images within which areas of low tracer uptake are conventionally interpreted as being ischaemic. Pharmacological vasodilatation, unlike exercise perfusion scintigraphy, produces an increase in coronary supply exceeding demand and areas of relative hypoperfusion may not necessarily be ischaemic. Furthermore, in clinical use, dipyridamole is seldom associated with obvious symptoms of myocardial ischaemia. ${ }^{78}$ Such findings have led to the suggestion that dipyridamole causes little, if any, myocardial ischaemia.

Development of transient asynergic wall motion in regions of the left ventricle is relatively specific in myocardial ischaemia.? 
This occurs in $50 \%$ to $60 \%$ of patients with significant coronary artery disease when given dipyridamole, and is evidence that myocardial ischaemia is at least partly a function of pharmacological coronary vasodilatation. ${ }^{1011}$ Physiological mechanisms for the occurrence of ischaemia have been put forward, ${ }^{12-14}$ but the true incidence of ischaemia with dipyridamole remains undefined. The definition of ischaemia within vasodilator perfusion imaging is important as the technique is gaining popularity and dipyridamole has been licensed for this purpose. Because dipyridamole produces perfusion deficits in regions of mild to moderate coronary stenosis pathophysiological importance of such lesions needs to be examined. ${ }^{15}$ We have used endocardial recordings of monophasic action potentials as an electrophysiological marker of localised ischaemia, to document the presence or absence of ischaemia in areas of perfusion deficits induced by dipyridamole. The duration of the monophasic action potentials measured under steady state conditions registers early changes of localised myocardial ischaemia, by shortening in response to ischaemia. ${ }^{16}{ }^{17}$ Recordings were made from the left, or right ventricular endocardium, or both during the administration of dipyridamole, and electrophysiological changes indicative of ischaemia were recorded in areas of myocardium with reversible perfusion defects as shown by technetium-99m hexakis-2methoxy-2-methypropyl-isonitrile (Tc-99m MIBI) and tomography.

\section{Patients and methods}

After routine cardiac catheterisation, monophasic action potentials from the ventricular endocardium were recorded during intravenous infusion of dipyridamole. When the drug was expected to exert maximal pharmacological effect (four minutes after dipyridamole infusion), Tc-99m MIBI was given for single photon emission computed

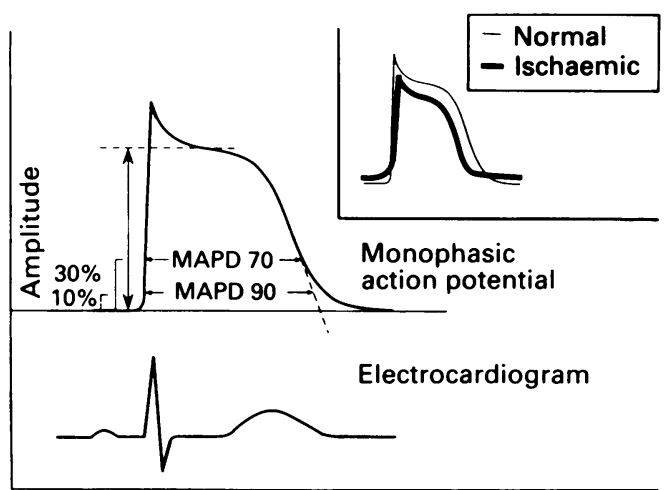

Figure 1 Typical monophasic action potential signal. The electrocardiogram is shown below the signal to indicate the temporal relation to the $O R S T$. The amplitude of the signal $(\mathrm{mV})$ is the distance between the diastolic baseline and the highest point on the plateau of the repolarisation phase. The duration of the action potential at $70 \%$ (MAPD 70 ) and $90 \%$ (MADP 90) repolarisation is measured with reference to the amplitude. The inset in figure 1 shows classic configurational changes in the action potential signal with ischaemia. tomographic imaging. The position of the catheter recording monophasic action potentials in the ventricle was found by biplane cinematography. Changes in the duration of the action potential were then related to the perfusion characteristics in the area of the recording.

\section{STUDY PATIENTS}

Thirty three patients undergoing routine cardiac catheterisation for investigation of chest pain were selected at random from the waiting list. Patients with unstable angina, atrial fibrillation, overt congestive heart failure, and obstructive airways disease were excluded from the study. Antianginal drugs were continued up to 12 hours before the study. Substances containing methyl xanthines were withheld for 24 hours before the study. Written informed consent was obtained from each patient and the study protocol was approved by our institution's ethical committee.

\section{ELECTROPHYSIOLOGICAL MEASUREMENTS}

Purpose built bioplar pressure contact catheters with silver and silver chloride electrodes (Cordis (UK) Ltd; 7 French size) were used to record monophasic action potentials. Apposition of the exploring electrode to the endocardial surface resulted in stable action potential signals with an amplitude of 20 to $40 \mathrm{mV}$. Details of signal collection and processing have been previously described. ${ }^{18}$

Figure 1 shows a typical monophasic action potential signal and its time relation to the QRST of the electrocardiogram. The upstroke of the action potential is equivalent to the $R$ wave and the downstroke (fast repolarisation phase) to the $T$ wave of the electrocardiogram. The amplitude of the signal is measured in $\mathrm{mV}$ as the distance between the diastolic baseline and the highest part of the plateau phase of repolarisation. Duration is measured in $\mathrm{ms}$ with reference to the total amplitude of the signal. Any alteration in amplitude of the signal therefore has little influence on the duration measurement as both values would change proportionately. The inset in fig 1 depicts configurational changes in the signal with ischaemia. Although ischaemia also produces a fall in total amplitude and upstroke velocity of the action potential, shortening of the duration is the most reliable index as the monophasic action potential reflects only a variable proportion of the amplitude and upstroke velocity of the intracellular action potential. ${ }^{19}$

\section{MYOCARDIAL SCINTIGRAPHY}

Tc-99m MIBI was used for myocardial perfusion scintigraphy with single photon emission tomography using a two day, two dose protocol. Images were acquired with a single detector rotating gamma camera and computer system (IGE $400 \mathrm{AC}-$ Starcam) equipped with a low energy, high resolution collimator. To get the images the following variables were used: a $20 \%$ energy window had a $3 \%$ offset around a $140 \mathrm{keV}$ technetium-99m photopeak; 64 half minute views were acquired over a $360^{\circ}$ circular arc of rotation starting at $45^{\circ}$ right anterior 
oblique; data acquisition and storage was on a $128 \times 128$ word matrix; acquired data was prefiltered with a Hanning filter $(0.75$ cycles/ $\mathrm{cm}$ ) and transformed to a $64 \times 64$ word matrix. A back projection reconstruction algorithm was used to get transaxial sections (one pixel thick). These reconstructed tomographic slices were then reoriented in the short axis, horizontal long, and vertical long axes for display and visual analysis.

\section{PROCEDURE}

Routine left ventricular and coronary angiography was performed through the femoral route by the Judkin's technique with a nonionic angiographic dye (Omnipaque 350 , Nycomed (UK) Ltd). This was followed by positioning of catheters, for the measurement of monophasic action potentials, in the left, or right ventricular endocardium, or both, through the femoral arterial or venous sheaths. A bipolar temporary pacing electrode was introduced into the right atrium through a second femoral venous sheath. Atrial pacing was established at $20 \%$ above the patient's resting heart rate to maintain a constant heart rate during the procedure.

Arterial blood pressure was monitored through the side arm of the femoral arterial cannula. The electrocardiogram with limb leads I and II and chest lead V5 was continously monitored. Monophasic action potentials were recorded from a single site in the left, or right ventricle, or both after pacing had been established for three minutes (to allow for adaptation of the duration of the action potentials). Dipyridamole was infused through a forearm vein at a rate of $0.142 \mathrm{mg} / \mathrm{kg} / \mathrm{min}$ over four minutes. Recordings of action potentials, arterial pressure, and electrocardiogram were made every minute for a total of 15 minutes.

Four minutes after completion of dipyridamole infusion, $370 \mathrm{MBq}$ of $\mathrm{Tc}-99 \mathrm{~m}$ MIBI was given intravenously. If any patient developed severe angina or haemodynamic upset, the effect of dipyridamole was reversed using 125 to $250 \mathrm{mg}$ of aminophylline given intravenously two minutes after Tc-99m MIBI. Patients were returned to the ward and encouraged to eat a light fatty meal to facilitate clearance of the isotope from the liver. Myocardial perfusion imaging with tomography was carried out between one and two hours after injection of the tracer. Rest imaging was performed 24 hours later after the patient had fasted for four hours and after a second dose of $370 \mathrm{MBq}$ of Tc-99m MIBI. Data collection was started one to two hours after injection following a light fatty meal.

\section{DATA ANALYSIS}

Coronary angiographic data were interpreted by visual inspection and the degree of stenosis was classed as mild, moderate, or severe. Regional collateral circulation was confirmed on the basis of retrograde filling of distal segments of totally or subtotally occluded arteries. Regions supplied by arteries with more than $50 \%$ stenosis (but non-occluded) and without angiographically obvious collateral circulation were designated areas without collateral vessels.

Myocardial perfusion images were analysed and interpreted by one observer blinded to the angiographic and monophasic action potential data. In case of equivocal results, a consensus opinion was accepted. Images were considered normal if they were without defect in the initial set or abnormal if there were reversible or fixed defects. Position of the defect was related to standard coronary anatomy where anterior and septal abnormalities were considered to be in the territory of the left anterior descending artery, lateral or posterolateral defects in the territory of the left circumflex artery, and inferior abnormalities in the territory of the right coronary artery. Apical defects could relate to the territory of any major coronary artery. ${ }^{20}$

The duration of the monophasic action potentials was measured by an observer blinded to the angiographic and scintigraphic data. Measurements were made at both $70 \%$ and $90 \%$ repolarisation. As both values registered a similar pattern, only values at $90 \%$ repolarisation are included in the text.

The position of the catheter measuring action potentials in the ventricle was documented by biplane cinematography at the time of cardiac catheterisation and was subsequently related to the perfusion abnormalities on the perfusion scan. Recordings were divided into three groups based on the relation of the catheter tip to areas of scintigraphic perfusion defects and coronary anatomical data for the action potential recording site; group 1 includes recordings from areas with a normal perfusion pattern; group 2 includes recordings from areas with reversible perfusion pattern but subtended by stenosed arteries without angiographically apparent collateral vessels; group 3 includes recordings from areas with reversible pattern but subtended by totally or subtotally occluded arteries with collateral filling of the distal vessel.

Statistical analysis of comparisons between groups were made by analysis of variance with planned comparison of the means (ANOVA). For graphical representation and to facilitate visual display, changes in duration of action potential were expressed as ratios: for each single site recording, the control value of duration of action potential was taken to represent $100 \%$ and any alteration was related to the control value. Pooled data are expressed as means (SEM).

\section{Results}

Of 33 patients studied, heart rate control during infusion of dipyridamole was lost in two patients and as the duration of action potential is dependent on cycle length, these two patients were excluded from the analysis. The results reported are on the 31 patients in whom steady state single site recordings of action potentials were obtained from the ventricles. The table gives patient details. Median age of the patients studied was 56 (range 33-72) years. All except two patients had significant coronary artery 
disease defined as greater than $50 \%$ stenosis in at least one major epicardial artery; 12 of these had single vessel disease, 12 two vessel disease, and five triple vessel disease. Previous aortocoronary bypass grafting had been performed in three patients.
SENSITIVITY OF MYOCARDIAL PERFUSION SCINTIGRAPHY

All patients with significant coronary artery disease showed perfusion abnormalities on imaging. Among 51 coronary arteries with significant stenoses, 44 showed related per-

Details of patients (all men) in the study

\begin{tabular}{|c|c|c|c|c|c|c|c|c|c|c|}
\hline \multirow{2}{*}{$\begin{array}{l}\text { Patient } \\
\text { no }\end{array}$} & \multirow[b]{2}{*}{ Age } & \multirow[b]{2}{*}{ Drugs } & \multicolumn{3}{|c|}{ Coronary artery anatomy } & \multirow{2}{*}{$\begin{array}{l}\text { Paced rate } \\
(b p m)\end{array}$} & \multirow{2}{*}{$\begin{array}{l}\text { Angina } \\
S T \text { change }\end{array}$} & \multirow[b]{2}{*}{ Perfusion scan } & \multirow{2}{*}{$\begin{array}{l}\text { MAP catheter } \\
\text { position }\end{array}$} & \multirow[b]{2}{*}{ Group } \\
\hline & & & $L A D$ & $L C X$ & $R C A$ & & & & & \\
\hline 1 & 54 & $\begin{array}{l}\text { Atenolol } \\
\text { Diltiaz }\end{array}$ & $\begin{array}{l}\text { Moderate } \\
\text { NC }\end{array}$ & $\begin{array}{l}\text { Moderate } \\
\text { NC }\end{array}$ & $\begin{array}{l}\text { Severe } \\
\text { NC }\end{array}$ & 80 & $\begin{array}{l}\mathbf{A P}+ \\
\mathbf{S T}_{-}\end{array}$ & $\begin{array}{l}\text { Inferior, lateral, } \\
\text { and anteroapical RPD, } \\
\text { septum normal }\end{array}$ & $\begin{array}{l}\text { Lateral wall of LV } \\
\mathrm{RV} \text { mid-septum }\end{array}$ & $\begin{array}{l}\text { LV-2 } \\
\text { RV-1 }\end{array}$ \\
\hline 2 & 44 & Atenolol & Normal & $\begin{array}{l}\text { Moderate } \\
\text { NC }\end{array}$ & $\begin{array}{l}\text { Occluded } \\
\text { C }\end{array}$ & 80 & $\begin{array}{l}\text { AP+ } \\
\text { ST - }\end{array}$ & $\begin{array}{l}\text { inferior FD } \\
\text { lateral RPD }\end{array}$ & $\begin{array}{l}\text { Lateral wall of LV } \\
\text { RV mid-septum }\end{array}$ & $\begin{array}{l}\text { LV-2 } \\
\text { RV-1 }\end{array}$ \\
\hline 3 & 58 & $\begin{array}{l}\text { Propran } \\
\text { Nifed } \\
\text { Nitrate }\end{array}$ & $\begin{array}{l}\text { Moderate } \\
\text { NC }\end{array}$ & $\begin{array}{l}\text { Moderate } \\
\text { NC }\end{array}$ & Occluded & 75 & $\begin{array}{l}\mathrm{AP}+ \\
\mathrm{ST}+\end{array}$ & inferior RPD & RV mid-septum & RV-1 \\
\hline 4 & 50 & $\begin{array}{l}\text { Atenolol } \\
\text { Diltiaz }\end{array}$ & $\begin{array}{l}\text { Severe } \\
\text { NC }\end{array}$ & Normal & $\begin{array}{l}\text { Occluded } \\
\text { C }\end{array}$ & 80 & $\mathbf{A P +}$ & $\begin{array}{l}\text { Inferior and } \\
\text { anteroseptal RPD }\end{array}$ & $\begin{array}{l}\text { Inferoposterior } \\
\text { wall of } \mathrm{LV}\end{array}$ & LV-3 \\
\hline 5 & 56 & $\begin{array}{l}\text { Atenolol } \\
\text { Nifed }\end{array}$ & Normal & Normal & Normal & 80 & $\begin{array}{l}\mathbf{A P}+ \\
\mathbf{S T}-\end{array}$ & Normal & RV mid-septum & RV-1 \\
\hline 6 & 62 & Atenolol & Normal & $\begin{array}{l}\text { Moderate } \\
\text { NC }\end{array}$ & $\begin{array}{l}\text { Occluded } \\
\text { C }\end{array}$ & 80 & $\begin{array}{l}\mathbf{A P}- \\
\mathbf{S T}-\end{array}$ & $\begin{array}{l}\text { Inferior FD, } \\
\text { lateral RPD }\end{array}$ & $\begin{array}{l}\text { Posterolateral wall } \\
\text { of } \mathrm{LV} ; \mathrm{RV} \text { mid-septum }\end{array}$ & $\begin{array}{l}\text { LV-2 } \\
\text { RV-1 }\end{array}$ \\
\hline 7 & 48 & Atenolol & $\begin{array}{l}\text { Moderate } \\
\text { NC }\end{array}$ & Normal & $\begin{array}{l}\text { Occluded } \\
\text { C }\end{array}$ & 80 & $\underset{\text { ST - }}{\mathbf{A P}+}$ & $\begin{array}{l}\text { Inferior and } \\
\text { antero apical RPD }\end{array}$ & Lateral wall of $\mathrm{LV}$ & LV-1 \\
\hline 8 & 35 & $\begin{array}{l}\text { Atenolol } \\
\text { Nitrate }\end{array}$ & $\begin{array}{l}\text { Severe } \\
\text { NC }\end{array}$ & Mild & Normal & 80 & $\begin{array}{l}\text { AP - } \\
\text { ST - }\end{array}$ & $\begin{array}{l}\text { Anterior and septal } \\
\text { RPD }\end{array}$ & $\begin{array}{l}\text { Lateral wall of LV } \\
\text { RV mid-septum }\end{array}$ & $\begin{array}{l}\text { LV-1 } \\
\text { RV-2 }\end{array}$ \\
\hline 9 & 68 & $\begin{array}{l}\text { Metop } \\
\text { Nifed }\end{array}$ & $\begin{array}{l}\text { Severe } \\
\text { NC }\end{array}$ & Normal & $\begin{array}{l}\text { Severe } \\
\text { NC }\end{array}$ & 80 & $\begin{array}{l}\text { AP- } \\
\text { ST - }\end{array}$ & $\begin{array}{l}\text { Anterior, septal, and } \\
\text { inferior RPD }\end{array}$ & Lateral wall of $\mathrm{LV}$ & LV-1 \\
\hline 10 & 59 & Atenolol & $\begin{array}{l}\text { Severe } \\
\text { NC }\end{array}$ & $\begin{array}{l}\text { Moderate } \\
\text { NC }\end{array}$ & Mild & 90 & $\begin{array}{l}\text { AP+ } \\
\text { ST - }\end{array}$ & $\begin{array}{l}\text { Anterior and septal } \\
\text { RPD }\end{array}$ & $\begin{array}{l}\text { Lateral wall of LV } \\
\text { RV mid-septum }\end{array}$ & $\begin{array}{l}\text { LV-1 } \\
\text { RV-2 }\end{array}$ \\
\hline 11 & 49 & Nil & Normal & $\begin{array}{l}\text { Severe } \\
\text { NC }\end{array}$ & Normal & 100 & $\begin{array}{l}\text { AP+ } \\
\text { ST - }\end{array}$ & Inferolateral RPD & $\begin{array}{l}\text { Lateral wall of LV } \\
\text { RV mid-septum }\end{array}$ & $\begin{array}{l}\text { LV-2 } \\
\text { RV-1 }\end{array}$ \\
\hline 12 & 55 & $\begin{array}{l}\text { Atenolol } \\
\text { Nifed }\end{array}$ & Mild & $\begin{array}{l}\text { Occluded } \\
\text { NC }\end{array}$ & $\begin{array}{l}\text { Non } \\
\text { Dominant }\end{array}$ & 80 & $\begin{array}{l}\mathrm{AP}+ \\
\mathrm{ST}-\end{array}$ & $\begin{array}{l}\text { Inferior wall } \\
\text { partial RPD }\end{array}$ & $\begin{array}{l}\text { Lateral wall of LV } \\
\text { RV mid-septum }\end{array}$ & $\begin{array}{l}\text { LV-1 } \\
\text { RV-1 }\end{array}$ \\
\hline 13 & 57 & Nifed & Mild & Occluded & Normal & 90 & $\begin{array}{l}\mathrm{AP}+ \\
\mathrm{ST}+\end{array}$ & $\begin{array}{l}\text { Inferior and } \\
\text { lateral RPD }\end{array}$ & $\begin{array}{l}\text { Lateral wall of LV } \\
\text { RV mid-septum }\end{array}$ & $\begin{array}{l}\text { LV -3 } \\
\text { RV-1 }\end{array}$ \\
\hline 14 & 50 & Atenolol & $\begin{array}{l}\text { Severe } \\
\text { NC }\end{array}$ & Mild & $\begin{array}{l}\text { Occluded } \\
\text { C }\end{array}$ & 90 & $\begin{array}{l}\mathrm{AP}+ \\
\mathrm{ST}+\end{array}$ & $\begin{array}{l}\text { Anteroseptal and } \\
\text { inferior RPD }\end{array}$ & $\begin{array}{l}\text { RV inferoposterior } \\
\text { septum }\end{array}$ & $R V-3$ \\
\hline 15 & 54 & $\begin{array}{l}\text { Atenolol } \\
\text { Nifed }\end{array}$ & Normal & Normal & Normal & 90 & $\begin{array}{l}\text { AP+ } \\
\text { ST - }\end{array}$ & Normal & $\begin{array}{l}\text { Lateral wall of LV } \\
\text { RV apex }\end{array}$ & $\begin{array}{l}\text { LV-1 } \\
\text { RV-1 }\end{array}$ \\
\hline 16 & 56 & $\begin{array}{l}\text { Atenolol } \\
\text { Nifedip }\end{array}$ & $\begin{array}{l}\text { Severe } \\
\text { NC }\end{array}$ & Normal & $\begin{array}{l}\text { Occluded } \\
\text { C }\end{array}$ & 80 & $\begin{array}{l}\mathrm{AP}+ \\
\mathbf{S T}-\end{array}$ & $\begin{array}{l}\text { Anteroseptal RPD, } \\
\text { inferior FD }\end{array}$ & $\begin{array}{l}\text { Lateral wall of LV } \\
\text { RV mid-septum }\end{array}$ & $\begin{array}{l}\text { LV-1 } \\
\text { RV-2 }\end{array}$ \\
\hline 17 & 50 & Nil & Mild & $\begin{array}{l}\text { Moderate } \\
\text { NC }\end{array}$ & $\begin{array}{l}\text { Severe } \\
\text { NC }\end{array}$ & 100 & $\begin{array}{l}\mathbf{A P +} \\
\mathbf{S T}-\end{array}$ & $\begin{array}{l}\text { Inferior and } \\
\text { lateral RPD }\end{array}$ & $\begin{array}{l}\text { Lateral wall of LV } \\
\text { RV mid-septum }\end{array}$ & $\begin{array}{l}\text { LV-2 } \\
\text { RV-1 }\end{array}$ \\
\hline 18 & 58 & Metop & $\begin{array}{l}\text { Poor graft } \\
\text { run off-NC }\end{array}$ & Normal & Normal & 80 & $\begin{array}{l}\text { AP - } \\
\text { ST - }\end{array}$ & $\begin{array}{l}\text { Anteroseptal } \\
\text { partial RPD }\end{array}$ & RV apical septum & $\mathrm{RV}-2$ \\
\hline 19 & 68 & Metop & $\begin{array}{l}\text { Occluded } \\
\text { C }\end{array}$ & $\begin{array}{l}\text { Occluded } \\
\text { C }\end{array}$ & Patent graft & 90 & $\begin{array}{l}\mathrm{AP}+ \\
\mathrm{ST}+\end{array}$ & $\begin{array}{l}\text { Anterior FD, } \\
\text { lateral wall RPD }\end{array}$ & Lateral wall of $\mathrm{LV}$ & LV-3 \\
\hline 20 & 57 & $\begin{array}{l}\text { Metop } \\
\text { Diltiaz }\end{array}$ & $\begin{array}{l}\text { Moderate } \\
\text { NC }\end{array}$ & Normal & Normal & 90 & $\begin{array}{l}\text { AP+ } \\
\text { ST - }\end{array}$ & $\begin{array}{l}\text { Anteroapical } \\
\text { RPD, septum normal }\end{array}$ & RV mid-septum & RV-1 \\
\hline 21 & 33 & Atenolol & $\begin{array}{l}\text { Moderate } \\
\text { NC }\end{array}$ & Normal & Normal & 80 & $\begin{array}{l}\text { AP+ } \\
\text { ST - }\end{array}$ & $\begin{array}{l}\text { Mild anteroapical } \\
\text { RPD }\end{array}$ & $\begin{array}{l}\text { LV septum } \\
\text { RV mid-septum }\end{array}$ & $\begin{array}{l}\text { LV-1 } \\
\text { RV-1 }\end{array}$ \\
\hline 22 & 59 & $\begin{array}{l}\text { Atenolol } \\
\text { Nifed }\end{array}$ & $\begin{array}{l}\text { Occluded } \\
\text { C }\end{array}$ & Normal & Normal & 100 & $\begin{array}{l}\mathrm{AP}+ \\
\mathrm{ST}-\end{array}$ & $\begin{array}{l}\text { Anterior and septal } \\
\text { RPD, inferior } \\
\text { partial RPD }\end{array}$ & $\begin{array}{l}\text { Lateral wall of LV } \\
\text { RV mid-septum }\end{array}$ & $\begin{array}{l}\text { LV-1 } \\
\text { RV-3 }\end{array}$ \\
\hline 23 & 54 & Atenolol & Occluded & Normal & $\begin{array}{l}\text { Moderate } \\
\text { NC }\end{array}$ & 80 & $\begin{array}{l}\text { AP+ } \\
\text { ST- }\end{array}$ & $\begin{array}{l}\text { Anterior, septal, } \\
\text { and inferior RPD }\end{array}$ & $\begin{array}{l}\text { Lateral wall of } \mathrm{LV} \\
\mathrm{RV} \text { anterior wall }\end{array}$ & $\begin{array}{l}\text { LV-1 } \\
\text { RV-1 }\end{array}$ \\
\hline 24 & 54 & Nil & $\begin{array}{l}\text { Occluded } \\
\text { C }\end{array}$ & $\begin{array}{l}\text { Occluded } \\
\text { C }\end{array}$ & Normal & 90 & $\begin{array}{l}\mathrm{AP}+ \\
\mathrm{ST}+\end{array}$ & $\begin{array}{l}\text { Anteroapical FD, } \\
\text { lateral wall RPD, } \\
\text { septum normal }\end{array}$ & $\begin{array}{l}\text { Lateral wall of LV } \\
\text { RV mid-septum }\end{array}$ & $\begin{array}{l}\text { LV -3 } \\
\text { RV-1 }\end{array}$ \\
\hline 25 & 46 & Diltiaz & Normal & $\begin{array}{l}\text { Occluded } \\
\text { C }\end{array}$ & $\begin{array}{l}\text { Non } \\
\text { Dominant }\end{array}$ & 80 & $\begin{array}{l}\text { AP+ } \\
\text { ST }+\end{array}$ & $\begin{array}{l}\text { Lateral wall RPD, } \\
\text { partial RPD in } \\
\text { inferoapical region }\end{array}$ & $\begin{array}{l}\text { Lateral wall of } \mathrm{LV} \\
\mathrm{RV} \text { posterior septum }\end{array}$ & $\begin{array}{l}\text { LV-3 } \\
\text { RV-3 }\end{array}$ \\
\hline 26 & 60 & $\begin{array}{l}\text { Atenolol } \\
\text { Diltiaz }\end{array}$ & Mild & Normal & $\begin{array}{l}\text { Severe } \\
\text { NC }\end{array}$ & 100 & $\begin{array}{l}\mathbf{A P}+ \\
\mathbf{S T}-\end{array}$ & $\begin{array}{l}\text { Inferior and apical } \\
\text { RPD }\end{array}$ & $\begin{array}{l}\text { Lateral wall of } \mathrm{LV} \\
\mathrm{RV} \text { apex }\end{array}$ & $\begin{array}{l}\text { LV-1 } \\
\text { RV-1 }\end{array}$ \\
\hline 27 & 48 & & $\begin{array}{l}\text { Moderate } \\
\text { NC }\end{array}$ & $\begin{array}{l}\text { Moderate } \\
\text { NC }\end{array}$ & $\begin{array}{l}\text { Occluded } \\
\text { NC }\end{array}$ & 110 & $\begin{array}{l}\text { AP- } \\
\text { ST - }\end{array}$ & $\begin{array}{l}\text { Anteroseptal FD, } \\
\text { inferior RPD }\end{array}$ & $\begin{array}{l}\text { Lateral wall of } \mathrm{LV} \\
\mathrm{RV} \text { apex }\end{array}$ & $\begin{array}{l}\text { LV-1 } \\
\text { RV-1 }\end{array}$ \\
\hline 28 & 72 & Nifed & Occluded & $\begin{array}{l}\text { Moderate } \\
\text { NC }\end{array}$ & $\begin{array}{l}\text { Occluded } \\
\text { C }\end{array}$ & 90 & $\stackrel{\mathbf{A P}+}{\mathbf{S T}_{+}}$ & $\begin{array}{l}\text { Anterseptal RPD, } \\
\text { inferior FD }\end{array}$ & RV mid-septum & RV-3 \\
\hline 29 & 66 & $\begin{array}{l}\text { Atenolol } \\
\text { Nifed }\end{array}$ & $\begin{array}{l}\text { Patent } \\
\text { graft }\end{array}$ & $\begin{array}{l}\text { Patent } \\
\text { graft }\end{array}$ & $\begin{array}{l}\text { Occluded } \\
\text { C }\end{array}$ & 90 & $\begin{array}{l}\text { AP - } \\
\text { ST - }\end{array}$ & $\begin{array}{l}\text { Inferior wall } \\
\text { partial RPD }\end{array}$ & RV mid-septum & RV-1 \\
\hline 30 & 59 & $\begin{array}{l}\text { Atenolol } \\
\text { Nifed }\end{array}$ & Normal & Normal & $\begin{array}{l}\text { Severe } \\
\text { NC }\end{array}$ & 70 & $\begin{array}{l}\text { AP - } \\
\text { ST - }\end{array}$ & $\begin{array}{l}\text { Inferior wall and } \\
\text { inferior septal RPD }\end{array}$ & $\begin{array}{l}\text { RV posteroinferior } \\
\text { septum }\end{array}$ & RV-2 \\
\hline 31 & 64 & $\begin{array}{l}\text { Atenolol } \\
\text { Nifed }\end{array}$ & $\begin{array}{l}\text { Severe } \\
\text { C }\end{array}$ & $\begin{array}{l}\text { Moderate } \\
\text { NC }\end{array}$ & $\begin{array}{l}\text { Occluded } \\
\text { C }\end{array}$ & 70 & $\begin{array}{l}\mathbf{A P}+ \\
\mathbf{S T}+\end{array}$ & $\begin{array}{l}\text { Inferior wall FD, } \\
\text { anteroseptal RPD }\end{array}$ & $\begin{array}{l}\text { High lateral wall } \\
\text { of LV; RV } \\
\text { mid-septum }\end{array}$ & $\begin{array}{l}\text { LV-1 } \\
\text { RV-3 }\end{array}$ \\
\hline
\end{tabular}




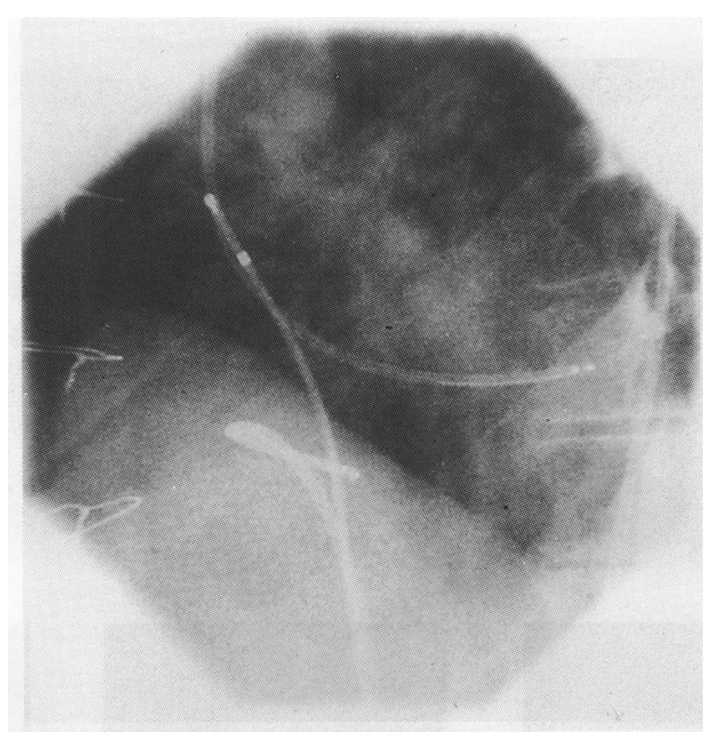

Figure 2 Typical catheter placements shown in the left anterior oblique view. Recording catheters were placed in the right and left ventricles. The right ventricular catheter has been manipulated on to the interventricular septum and in the left ventricle the catheter has been left in contact with the endocardium of the lateral wall. $A$ temporary pacing catheter is shown in the right atrium.

fusion abnormalities on scintigraphy thus yielding a per vessel sensitivity of $86 \cdot 3 \%$. When fixed defects due to previous myocardial infarction were excluded, the sensitivity fell to $83 \cdot 7 \%$.

\section{MONOPHASIC ACTION POTENTIAL RECORDINGS}

Figure 2 shows typical catheter placements during recording of action potentials. Fifty single site recordings were obtained from 31 patients (simultaneous recordings from right and left ventricles in 19 patients, the left ventricle alone in four patients, and right ventricle alone in eight patients). Based on the perfusion characteristics of the recording site, 30 recordings were assessed to be from normally perfused areas (group 1). Of the 20 recordings from abnormally perfused areas, 10 were from areas without collateral vessels (group 2), and 10 from areas with collaterals (group 3). Although the pacing rates used in each set of recordings varied, there was no

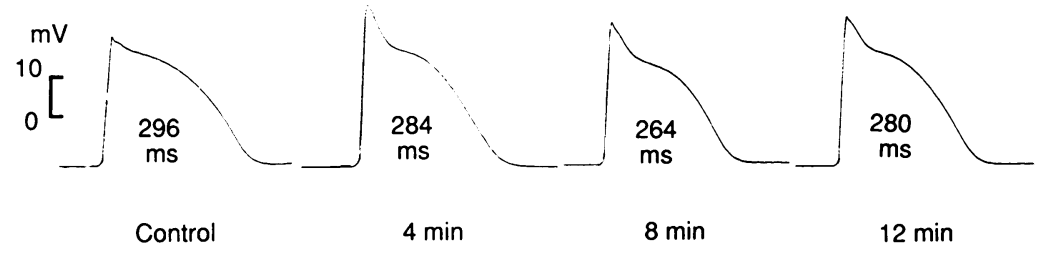

Figure 3 Serial steady state monophasic action potential signals recorded from the lateral left ventricular wall in patient 19 with occlusion of the native vessels and grafts to the territory of the left coronary circulation. This territory was perfused by collateral vessels from the right coronary artery and graft. Dipyridamole infusion produced noticeable shortening of the action potential duration, which becomes apparent four minutes from the start of dipyridamole infusion. Peak changes occur at eight minutes minutes from the start of dipyridamole infusion. Peak changes occur at eight minutes
when the action potential duration shortens by $32 \mathrm{~ms}$ compared with the control value. Regression of these changes is seen at 12 minutes, in this case enhanced by the administration of $125 \mathrm{mg}$ of aminophylline given at 10 minutes. Duration of monophasic action potentials in ms measured at $90 \%$ repolarisation (MADP 90 ) is shown within each action potential signal. significant difference in the paced heart rates between the three groups (mean (SE) values: group $187 \cdot 8(1 \cdot 9)$, group $284(3 \cdot 0)$, and group 3 $86(2 \cdot 7)$; NS).

Shortening of the duration of the action potential occurred in eight of the 10 recordings in group 2 and all recordings in group 3. Maximal changes were apparent eight minutes from the start of dipyridamole infusion. Figure 3 shows an example of the development and regression of the duration of the action potential that has shortened indicating ischaemia. Patient 19 had occlusion of native vessels and grafts to the territory of the left coronary circulation. Vessels from the right coronary artery and graft had formed collaterals to the territory of the left coronary circulation. Dipyridamole infusion produced considerable angina and ischaemic changes in recordings of monophasic action potentials from the lateral wall of the left ventricle. Shortening of the duration of the action potential was maximal at eight minutes. Regression of changes was apparent at 12 minutes, in this case acclerated by $125 \mathrm{mg}$ of aminophylline at 10 minutes.

\section{ILLUSTRATIVE STUDIES}

Figures 4 and 5 show coronary angiographic appearances, tomographic myocardial perfusion images, and typical examples of changes in monophasic action potential in studies representative of the three groups. Figures 4 A-D are data on patient 11 in table 1 . Coronary angiography shows normal right coronary and left anterior descending arteries producing a normal perfusion pattern in areas subtended by these arteries. The left main circumflex artery has a tight lesion without collateral supply. Perfusion images show reversible perfusion abnormalities in the lateral wall of the left ventricle. Monophasic action potentials recorded from the normally perfused septum show no change in the duration of the signals whereas those from the abnormally perfused lateral wall of the ventricle show shortening of the duration by $16 \mathrm{~ms}$. Figures $5 \mathrm{~A}-\mathrm{D}$ are from patient 22 . The left anterior descending artery is occluded at its origin. The region has collateral vessels fed by the right coronary artery. A large area of defective perfusion in the anterior and septal regions is produced with dipyridamole which partially reverses on the rest images. The duration of the action potentials recorded from the right ventricular septum show shortening by $40 \mathrm{~ms}$ whereas those from a normally perfused left ventricular lateral wall remain unchanged.

\section{ANALYSIS OF POOLED DATA}

The duration of the monophasic action potentials measured at $90 \%$ repolarisation altered from a control mean (SEM) of $276 \cdot 5(5 \cdot 3) \mathrm{ms}$ to $276 \cdot 6(5 \cdot 4) \mathrm{ms}$ for group 1 (NS), $289 \cdot 6$ (4.7) $\mathrm{ms}$ to $278.4(4.9)$ for group 2 recordings $(\mathrm{p}<0.001)$ and $269.6(5.7) \mathrm{ms}$ to $242.0(4.4)$ for group 3 recordings $(p<0.0001)$. Figure 6 shows the changes in action potential compared with the control values during dipyridamole infusion in the three groups. Significant ischaemia is apparent in the group 2 recordings 

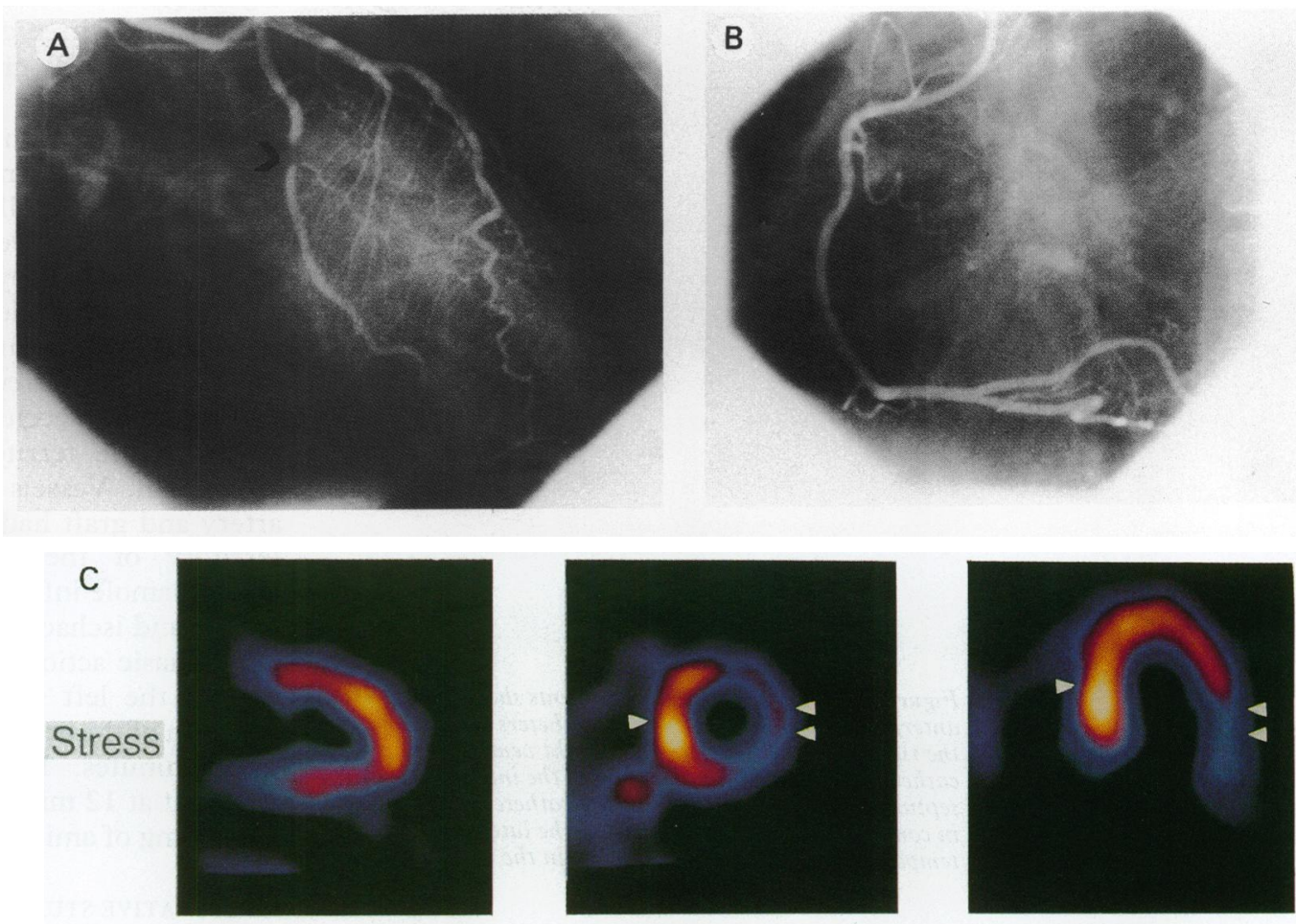

VLA

SA

HLA
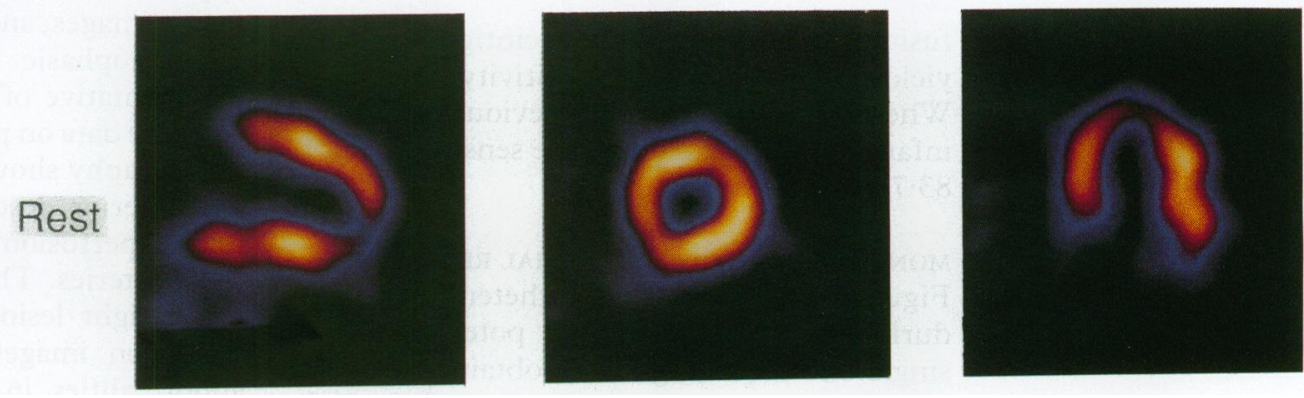

D

$$
{ }_{0}^{\mathrm{mV}}[
$$
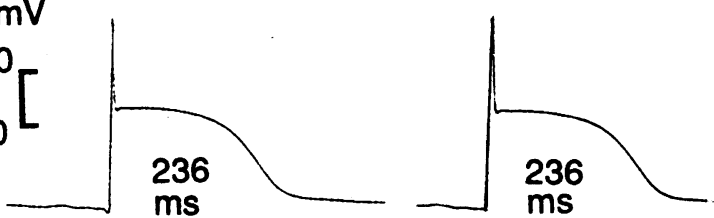

RV (Anterior septum)
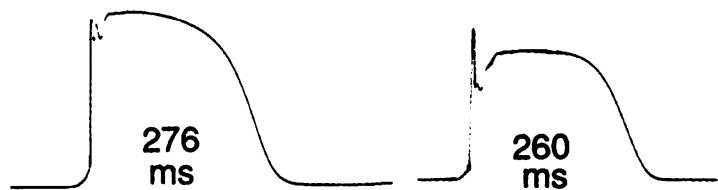

LV (Lateral wall)

Control

Peak (8 min)

Figure 4 Coronary angiographic appearances, myocardial perfusion images (single photon emission tomography), and monophasic action potential signals at control and at peak effects of dipyridamole for patient 11. Figure $4 A$ and $B$ show a tight stenosis (arrow) in the proximal course of the left circumflex artery without obvious collateral supply to the area subtended by this artery. Figure $4 C$ shows perfusion images with considerable lateral wall hypoperfusion during

dipyridamole stress which reperfuses with rest. Figure $4 D$ shows monophasic action potentials recorded simultaneously from the right ventricular mid-septum and left ventricular lateral wall (recording sites represented on the scintigram are indicated: single arrow for right ventricular septum and double arrows for left ventricular lateral wall). Duration of steady state action potential measured at $90 \%$ repolarisation (shown within signals) remains unchanged from the control value of the recording from the normally perfused septum whereas that for the area without collateral vessels but with abnormally perfused left ventricular lateral wall shortens by $16 \mathrm{~ms}$. VLA, vertical long axis; $S A$, short axis; wLA, horizontal long axis. 

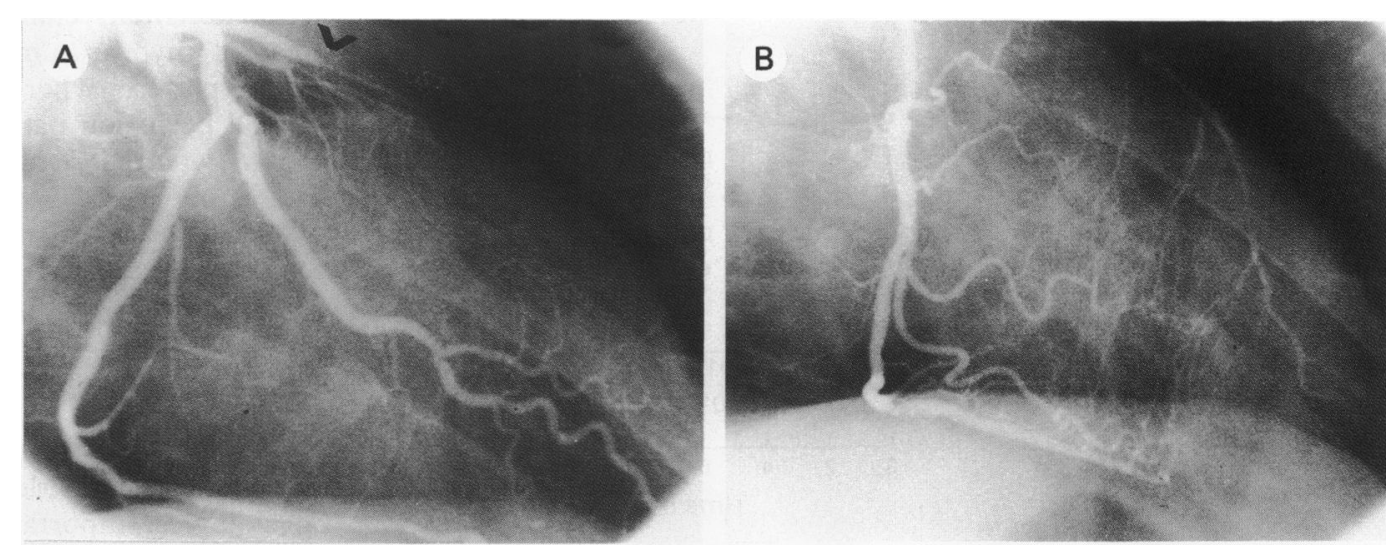

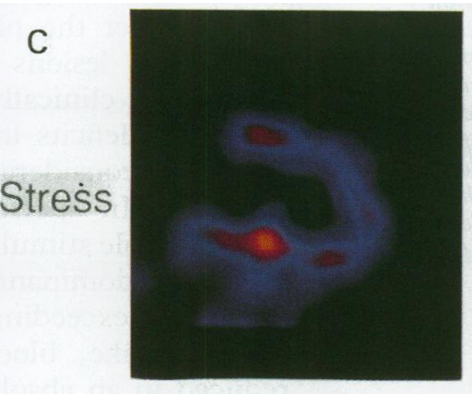

VLA

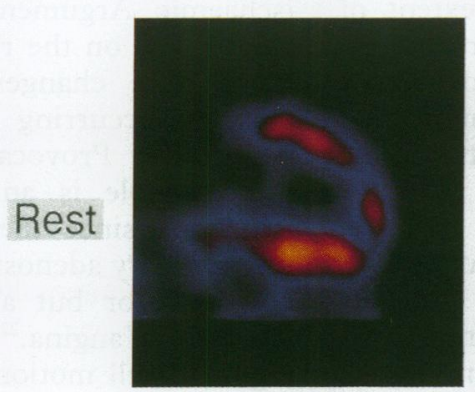

D

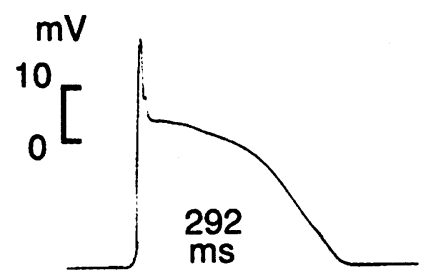

ms

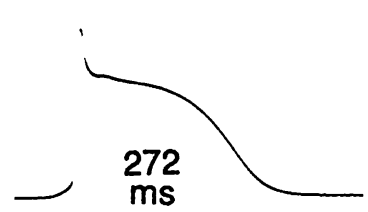

Control

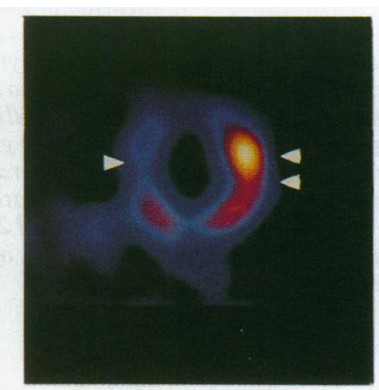

SA
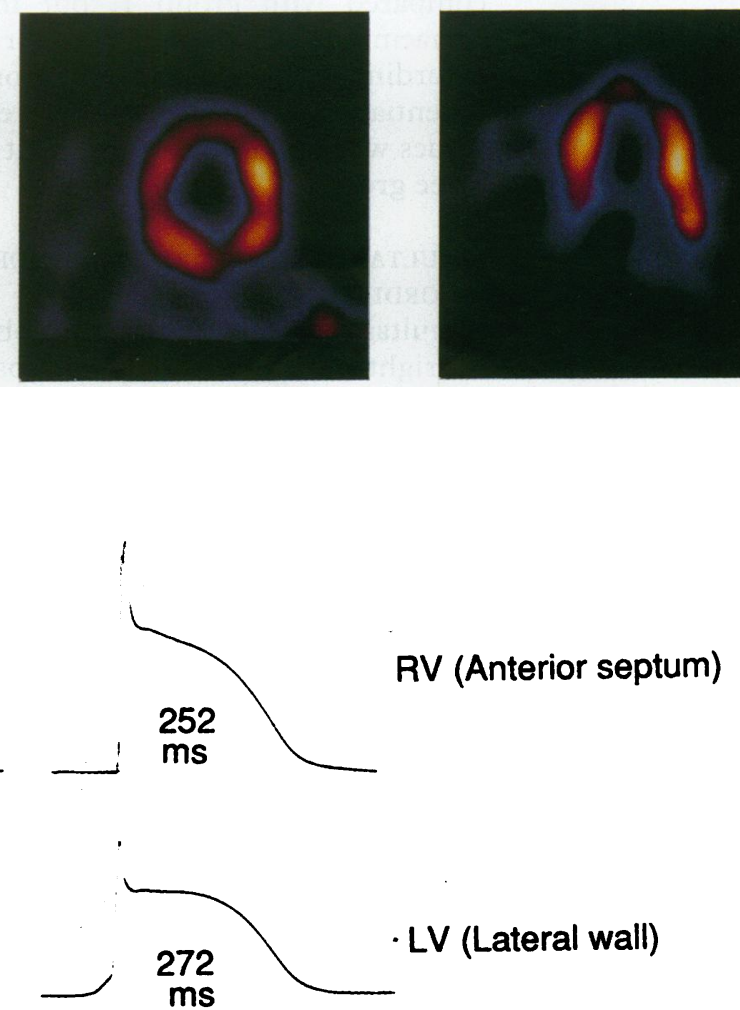

272

$\mathrm{ms}$

Peak (8 min)

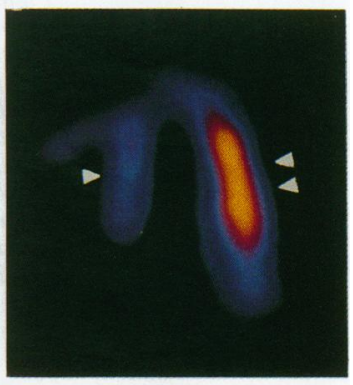

HLA

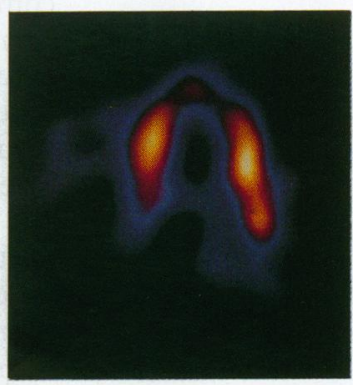

미

\}

Figure 5 Coronary angiographic appearances, myocardial perfusion images (single photon emission tomography), and momophic action potential signals at control and at peak dipyridamole effect for patient 22. Figure 5 shows occlusion of the left anterior descending artery at its origin $(A)$ arrow). The distal vessel is filled retrogradely by extensive collaterals from the normal right coronary artery (B). Perfusion images (figure 5C) show anterior, apical, and septal hypoperfusion during dipyridamole stress with partial reperfusion of these areas during rest. Figure $5 D$ shows simultaneus action potentials recorded from the right ventricular septum and the left ventricular lateral wall

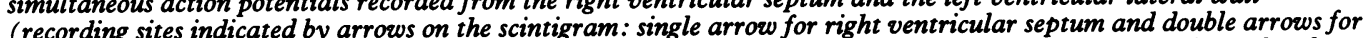
(recording sites indicated by arrows on the scintigram. single arrowfial of the right ventricle (abnormally perfused area left ventricular lateral wall). Duration of steady state action potential of the right ventricle (abnormally perfused area
with collateral vessels) measured at $90 \%$ repolarisation is 40 ms shorter than recordings from the normally perfused left with collateral vessels) measured at $90 \%$ repolarisation is 40 ms shorter than recordings from the normally perfused left
ventricular lateral wall that show no alteration in the duration from control value (duration of action potentials shown within signals). VLA, vertical long axis; $S A$, short axis; $H L A$, horizontal long axis. 


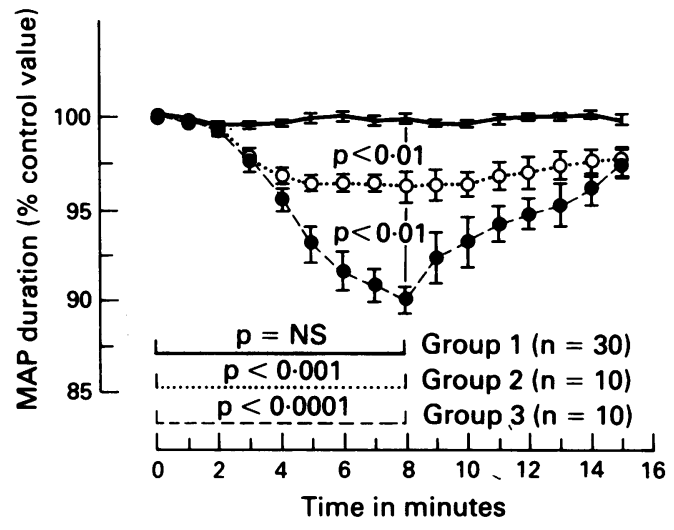

Figure 6 Mean changes in duration of the action potentials of the three groups are shown as a percentage of the control values. During infusion of dipyridamole, the group 1 (normal zone recordings) show no change. Group 2 (abnormal zone without collateral vessels) recordings shorten significantly when compared with group 1 . The changes are maximal for group 3 (collateral blood flow in an abnormal zone) with significantly greater shortening of the action potentials than in groups 1 and 2. Peak changes are apparent eight minutes after the start of dipyridamole administration.

ischaemia is apparent in the group 2 recordings compared with group 1, but the extent of ischaemia is substantially greater for group 3 recordings (changes in duration of action potentials from control to the eight minute values were significantly different between the three groups $\mathrm{p}<0.01$ ).

\section{SIMULTANEOUS NORMAL AND ABNORMAL ZONE RECORDINGS}

Simultaneous recordings were obtained from the right and left ventricles in 18 patients. In 12 of these, recordings were obtained from one normally and one abnormally perfused site. Mean (SEM) change in the duration of action potentials in ms between control values and eight minute values were as follows: $276 \cdot 3$ $(10 \cdot 2) v 276 \cdot 0(10 \cdot 3)$ for group $1(\mathrm{n}=12 ; \mathrm{NS})$; $287.5(5.6) v 274.0(4.9)$ for group $2(\mathrm{n}=8$; $\mathrm{p}<0.0006) ; 277.0(8.7) v 244(8.5)$ for group 3 $(\mathrm{n}=4 ; \mathrm{p}<0.001)$. These changes were significantly different between the three groups $(\mathrm{p}<0.01)$.

\section{ANGINA AND ST SEGMENT CHANGES WITH} INFUSION OF DIPYRIDAMOLE

Twenty four of the 31 patients developed angina during infusion of dipyridamole. This included the two patients with angiographically normal coronary arteries and normal perfusion images. ST segment depression was evident in only eight patients, all with areas of viable myocardium perfused by collateral vessels.

\section{Discussion}

There has been inconclusive evidence to settle the question whether transient defects detected by perfusion scintigraphy with dipyridamole stress represent ischaemia or merely redistribution of blood flow. This study has shown shortening of the duration of monophasic action potentials, characteristic of ischaemia, in areas of myocardium with a reversible perfusion pattern whereas in areas showing a normal perfusion pattern, the duration of the action potential remained unchanged. These data support the view that some degree of ischaemia is usually provoked by selective coronary vasodilation where there is significant flow limiting stenosis. Ischaemia was most evident in areas subtended by occluded arteries with distal collateral circulation from adjacent vessels.

Coronary angiography provides anatomical information with little indication of the pathophysiological importance of coronary stenoses. Perfusion scintigraphy on the other hand, could depict the physiological equivalent of anatomical lesions and therefore be more meaningful clinically. This may hold true for perfusion deficits induced by exercise where there is considerable increase in oxygen demand. In perfusion scintigraphy with dipyridamole stimulus this may not be the case as the predominant mechanism of action is blood flow exceeding demand. In areas of low tracer uptake, blood flow is therefore not reduced in an absolute sense, and such areas should not be considered necessarily ischaemic. Arguments against ischaemia have been based on the relative lack of electrocardiographic changes during dipyridamole infusion occurring in only 20 to $30 \%$ of patients. ${ }^{21}$ Provocation of angina with dipyridamole is an unreliable indicator of ischaemia since the drug acts through the intermediary adenosine. Adenosine is not only a vasodilator but also a messenger for the sensation of angina. ${ }^{22}$ Nevertheless, asynergy of regional wall motion occurs in some patients given intravenous dipyridamole. ${ }^{101123}$ This would suggest that flow differences induced by the drug are of sufficient magnitude to provoke myocardial ischaemia leading to transient contractile dysfunction. Our study, with an independent marker, provides good evidence for ischaemia being a significant component of dipyridamole perfusion imaging.

At present, practical methods of identifying localised ischaemia in clinical studies with dipyridamole are the surface electrocardiogram and wall motion asynergy found by any of the imaging techniques. The surface electrocardiogram is relatively insensitive to early myocardial ischaemia and not readily applicable to the interpretation of regional myocardial ischaemia. ${ }^{18}$ Although wall motion abnormalities are known accompaniments of early ischaemia, they have been documented in only 50 to $60 \%$ of patients with significant coronary artery disease when given a coronary vasodilator. ${ }^{114}$ This may be due to the relative insensitivity of the currently available imaging techniques to discern small changes that result from early subendocardial myocardial ischaemia. We therefore chose the monophasic action potential signal that records from an area of myocardium about $5 \mathrm{~mm}$ in diameter and is therefore suitable for exploring localised electrophysiological events.

Monophasic action potentials recorded from 
the surface of the myocardium reflect an averaged intracellular action potential for the group of cells beneath the exploring electrode. ${ }^{24}$ The amplitude of the signal is less than the intracellular action potential but the duration is reliable for the entire repolarisation phase. ${ }^{192526}$ Ischaemia shortens the duration of the action potential by mechanisms that are probably multifactorial. ${ }^{27}$ Recordings of monophasic action potentials have been used to monitor ischaemia on both endocardial and epicardial surfaces in animals and in humans. ${ }^{16172829}$ In endocardial studies, the ability of these recordings to detect early changes of ischaemia, which are typically localised to small areas, may be reduced by the conductor properties of blood within the cavity. The exploring electrode is in direct contact with the endocardium. The reference electrode situated $5 \mathrm{~mm}$ behind the catheter tip makes contact with the endocardium through the blood in the cavity. Solid angle theory thus predicts that a large area of ischaemia would be relatively undiluted by more distant contact with the surrounding normal myocardium. ${ }^{30}$ A small area of ischaemia on the other hand would subtend only a small angle to the reference electrode which might be influenced to a greater extent by the surrounding normal myocardium. In our studies, any error would be an underestimation of the severity of ischaemia.

The shortening of duration of action potentials found is likely to have been a direct result of ischaemia rather than changes related to alterations in catecholamine and potassium concentrations. Neither of these chemicals vary in concentration during the course of the protocol we used (unpublished observations). Dipyridamole would be expected to produce increased myocardial concentrations of adenosine, but adenosine has no significant effect on the duration of ventricular action potentials. ${ }^{31}$ Furthermore, in the patients in whom simultaneous recordings were obtained from abnormally and normally perfused areas, the duration of action potentials remained stable throughout the entire 15 minutes of recording in the normally perfused areas.

Most patients in this study were taking $\beta$ receptor blockers or calcium channel blockers. Neither of these agents are conventionally recognised as influencing the duration of ventricular action potentials. ${ }^{32}$ Antianginal medications reduce the ischaemic response to dipyridamole without limiting the hyperemic flow pattern induced by the drug. ${ }^{14}$ The number of patients not taking any medication was too few to allow meaningful comparison and assessment of the influence of these drugs on the extent of ischaemia. Nevertheless, we consider it unlikely that either group of medications would have encouraged the development of ischaemia.

The importance of this study is that we have provided strong evidence for the presence of some ischaemia as an integral part of vasodilator myocardial perfusion imaging. Particularly interesting was the striking difference between recordings of action potentials from ischaemic areas showing collateral vessels on angiography compared with areas without collaterals. Collateral circulation in humans develops in response to stimuli arising from prolonged periods of blood flow limitation, and possibly depends on genetically mediated angiogenic factors. ${ }^{33} 34$ These vessels are generally considered protective to compromised areas of myocardium. In the context of local coronary vasodilators, however, the reverse would seem to be the case. Such deleterious effects of epicardial collateral vessels have been suggested in previous reports. ${ }^{35}{ }^{36}$ The likely mechanism would be a local coronary steal because of a relative stenosis at the junction of the feeding vessel with the origin of the collaterals. ${ }^{33}$

The high incidence of ischaemia in this study is contrary to previous studies examining the question of ischaemia with coronary vasodilation in coronary artery disease. ${ }^{153738}$ These previous studies have focused on electrocardiographic and wall motion abnormalities as indicators of ischaemia. Whereas profound wall motion changes are known to occur a few beats after major coronary artery occlusion in experimental studies, the situation with regard to local subendocardial ischaemia induced by a coronary steal may be different and may not produce discernible wall motion abnormalities. An endocardial to epicardial blood flow steal induced by dipyridamole has been suggested by other investigators ${ }^{149}$ and is the probable mechanism in the genesis of the ischaemia for areas without collateral vessels in this study. It is nevertheless unlikely that such early ischaemia contributes significantly to the differential uptake of radiotracers in vasodilator perfusion imaging. Most perfusion abnormalities that are commonly seen with vasodilation would be more in keeping with redistribution as the primary mechanism. Ischaemia appears to be provoked in the course of such redistribution of blood flow especially when myocardial viability is dependent upon collateral vessels.

\section{Conclusion}

In this study we set out to investigate whether the reversible perfusion defects induced by dipyridamole and seen on myocardial perfusion scintigraphy was associated with the presence of myocardial ischaemia. We would interpret our results as indicating that where there is significant flow limiting coronary stenosis some degree of ischaemia is almost always provoked in the course of redistribution of the coronary flow induced by dipyridamole. Such ischaemia is of greater intensity in areas of viable myocardium with collateral supply. These findings have important implications in the physiological interpretation of a diagnostic test that is rapidly gaining popularity.

Dr John was supported by a grant from the special trustees of the Middlesex Hospital, London.

1 Gould KL, Westcott RJ, Albro PC, Hamilton GW. Noninvasive assessment of coronary stenoses by vasodilation: II Clinical methodology and feasibility. $\mathrm{Am} \mathrm{J}$ cardiol 1978;41:279-87. 
2 Boucher CA, Brewster DC, Darling RC, Okada RD, Strauss HW, Pohost GM. Determination of cardiac risk by dipyridamole-thallium imaging before peripheral vascular surgery. $N$ Engl J Med 1985;312:389-94.

3 Verani MS, Mahmarian JJ, Hixson JB, Boyce TM, Straudacher RA. Diagnosis of coronary artery disease by controlled coronary vasodilation with adnosine and challium-201 scintigraphy in patients unable to exercise. Circulation 1990;82:80-7.

4 Strauss HW, Pitt B. Non invasive detection of subcritical coronary artery narrowings with a coronary vasodilator and myocardial

5 Sorensen SG, Groves BM, Horwitz LD, Chaudhuri TK. Regional myocardial blood flow in man during dipyridamole coronary vasodilation. Chest $1985 ; 87: 735-9$.

6 Klocke FJ. Measurement of coronary flow reserve: defining pathophysiology versus making decisions about patien care. Circulation 1987;76:1183-9.

7 Beller G. Dipyridamole thallium-201 scintigraphy: an excellent alternative to exercise scintigraphy. $\mathrm{J} \mathrm{Am} \mathrm{Col}$ Cardiol 1989;14:1642-4.

8 Ranhosky A, Kempthorne-Rawson J, and the Intravenous Dipyridamole Thallium Imaging Study Group. The safety of intravenous dipyridamole thallium myocardial perfusion imaging. Circulation 1990;81:1205-9.

9 Beller G, Gibson RS. Sensitivity, specificity and prognostic significance of non-invasive testing for occult or known

10 Picano E, Distante A, Masini M, Morales MA, Lattanzi F, L'Abbate A. Dipyridamole-echocardiography test in L'Abbate A. Dipyridamole-echocardiography te

11 Pennell DJ, Underwood SR, Ell PJ, Swanton RH, Walker JM, Longmore DB. Dipyridamole magnetic resonance imaging; a comparison of thallium-201 emission tomography. Br Heart J 1990;64:362-9.

12 Flameng $W$, Wusten $B$, Schaper $W$. On the distribution of myocardial blood flow II. Effects of arterial stenosis and vasodilation. Basic Res Cardiol 1974;69:435-46.

13 Keltz TN, Innerfield M, Gitler B, Cooper JA. Dipyridamole induced myocardial ischaemia. JAMA 1987;257:1515-6.

14 Picano E. Dipyridamole - echocardiography test: historical background and physiologic basis. Eur Heart $J 1989$ 10:365-76.

15 Josephson MA, Brown G, Hecht HS, Hopkins J, Pierce CD Peterson RB. Noninvasive detection and localization of coronary stenosis in patients: comparison of resting, dipyridamole and exercise thallium-201 myocardial perfusion imaging. Am Heart $J$ 1982;103:1008-18.

16 Franz MR, Flaherty JT, Platia EV, Bulkley BH, Weisfeld ML. Localisation of regional myocardial ischaemia by ML. Localisation of regional myocardial ischaemia by recording mono

17 Taggart $P$, Sutton $P$, Runnalls $M, O$ 'Brien W, Donaldson $R$ Hayward $R$, et al. Use of the monophasic action potentia recording during routine coronary artery bypass surgery as an index of localised myocardial ischaemia. Lance 1986;i:1462-5.

18 Taggart $P$, Sutton $P$, John R, Hayward R, Swanton $H$. The epicardial electrogram: a quantitative assessment during balloon angioplasty incorporating monophasic action potential recordings. Br Heart J 1989;62:342-52.

19 Hoffman BF, Cranefield PF, Lepeschkin E, Surawicz B, Herrlich HC. Comparison of cardiac monophasic action potential recorded by intracellular and suction electrodes. Am J Physiol 1959;196:1297-301.

20 Borges-Neto S, Mahmarian JJ, Jain A, Roberts R, Veran MS. Quantitative thallium-201 single photon emission computed tomography after oral dipyridamole for assessing the presence, anatomic location and severity of coroning the presence, anatomic location and severity of coro

21 Zhu YY, Lee W, Botvinick E, Dae M, Chatteriee $K$ Danforth J, Ports T. The clinical and pathophysiologic implications of pain, ST abnormalities and scintigraphic changes during dipyridamole infusion: their relationship to peripheral haemodynamic response. Am Heart 1988;116:1071-80.

22 Lagerquist B, Sylven C, Beerman B, Helmins G, Waldenström $A$. Intracoronary adenosine causes angina pectoris like pain-an inquiry into the nature of visceral pain. Cardiovasc Res 1990;24:609-13.

23 Sochor H, Pachinger O, Ogris E, Probst P, Kaindl F. Radionuclide imaging after coronary vasodilation: myocardial scintigraphy with thallium-201 and radionuclide angiography after administration of dipyridamole. Eur Heart $J$ 1984;5:500-9.

24 Olsson SB Brorson L Edvardsson N, Varnauskas E Estimation of ventricular repolarisation in man by monophasic action potential recording technique. Eur Heart $J$ 1985;6(suppl D):71-9.

25 Ino T, Karaguezian HS, Hong K, Meesman M, Mandel WJ Peter $T$. Relation of monophasic acton potential recorded with contact electrode to underlying transmembrane action potential properties in isolated cardiac tissue: a systemic microelectrode validation study. Cardiovasc $R e s$ 1988;22:255-64.

26 Franz MR, Burkhoff D, Spurgeon H, Weisfeldt ML, Lakatta EG. In vitro validation of a new cardiac catheter technique for recording monophasic action potentials. Eur Heart 1986;7:34-41.

27 Janse MJ, Wit AL. Electrophysiological mechanisms of ventricular arrhythmias resulting from myocardial ventricular arrhythmias resulting from myocardial

28 Donaldson RM, Taggart P, Nashat F, Abed J, Rickards AF Noble D. Study of electrophysiological effects of early or Nubendocardial ischaemia with intracavitary electrodes in subendocardial ischaemia with in

29 Donaldson RM, Taggart P, Bennet JG, Rickards AF. Study of electrophysiological ischaemic events during coronary angioplasty. Texas Heart Journal 1984;11:24-30.

30 Holland RP, Arnsdorf MF. Solid angle theory and the electrocardiogram: physiologic and quantitative interpretations. Prog Cardiovasc Dis 1977;19:431-57.

31 Nunain SO, Garratt C, Paul V, Debbas NMG, Ward DE Camm AJ. The effect of intravenous adnosine on atrial and ventricular repolarisation. [Abstract]. J Am Coll Cardio 1991;17:39A.

32 Vaughan Williams EM. A classification of antiarrhythmic actions reassessed after a decade of new drugs. $J$ Clin Pharmacol 1984;24:129-47.

33 Gould KL. Coronary steal-is it clinically important? Chest 1989;96:227-8.

34 Kurachi K, Davie EW, Strydom DJ, Riordan JF, Vallee BL. Sequence of $\mathrm{cDNA}$ and gene for angiogenin, a human Sequence of cDNA and gene for angiogenin, a

35 Demer LL, Goldstein R, Mullani L, Kirkeeide R, et al. Coronary steal by non invasive PET identifies collateralised myocardium [abstract]. J Nucl Med 1986;27:977.

36 Chambers CE, Brown KA. Dipyridamole-induced ST seg ment depression during thallium-201 imaging in patient with coronary artery disease: angiographic and haemodynamic determinants. J Am Coll Cardiol 1988; 12:37-41.

37 Leppo J, Boucher CA, Okada RD, Newell JB, Strauss HW Pohost GM. Serial thallium-201 myocardial imaging after dipyridamole infusion; diagnostic utility in detecting motion. Circulation 1982;66:649-57.

38 Okada RD, Bendersky R, Strauss W, Pohost GM, Boucher C. Comparison of intravenous dipyridamole thallium cardiac imaging with exercise radionuclide angiography. Am Heart J1987;114:524-31.

39 Meerdink DJ, Okada RD, Leppo JA. The effect of dipyridamole on transmural blood flow gradients. Chest dipyridamole on 